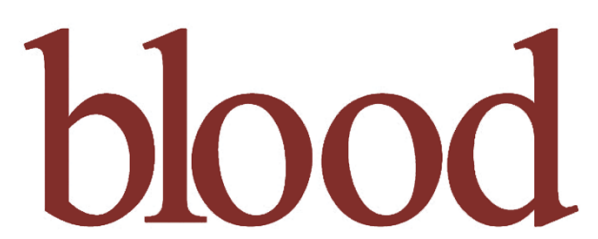

2005 105: 4247-4254

Prepublished online Feb 22, 2005;

doi:10.1182/blood-2004-11-4564

\title{
An inducible caspase 9 safety switch for T-cell therapy
}

Karin C. Straathof, Martin A. Pulè, Patricia Yotnda, Gianpietro Dotti, Elio F. Vanin, Malcolm K. Brenner, Helen E. Heslop, David M. Spencer and Cliona M. Rooney

Updated information and services can be found at:

http://bloodjournal.hematologylibrary.org/cgi/content/full/105/11/4247

Articles on similar topics may be found in the following Blood collections:

Apoptosis (747 articles)

Immunotherapy (577 articles)

Gene Therapy (389 articles)

Immunobiology (3565 articles)

Information about reproducing this article in parts or in its entirety may be found online at:

http://bloodjournal.hematologylibrary.org/misc/rights.dtl\#repub_requests

Information about ordering reprints may be found online at:

http://bloodjournal.hematologylibrary.org/misc/rights.dtl\#reprints

Information about subscriptions and ASH membership may be found online at:

http://bloodjournal.hematologylibrary.org/subscriptions/index.dtl

Blood (print ISSN 0006-4971, online ISSN 1528-0020), is published semimonthly by the American Society of Hematology, 1900 M St, NW, Suite 200, Washington DC 20036.

Copyright 2007 by The American Society of Hematology; all rights reserved.

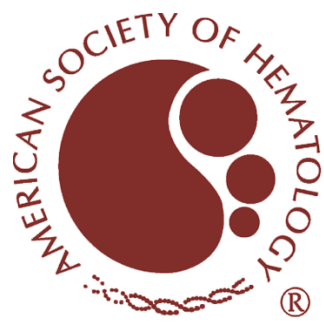




\title{
An inducible caspase 9 safety switch for T-cell therapy
}

Karin C. Straathof, Martin A. Pulè, Patricia Yotnda, Gianpietro Dotti, Elio F. Vanin, Malcolm K. Brenner, Helen E. Heslop, David M. Spencer, and Cliona M. Rooney

\begin{abstract}
The efficacy of adoptive T-cell therapy as treatment for malignancies may be enhanced by genetic modification of infused cells. However, oncogenic events due to vector/transgene integration, and toxicities due to the infused cells themselves, have tempered enthusiasm. A safe and efficient means of removing aberrant cells in vivo would ameliorate these concerns. We describe a "safety switch" that can be stably and efficiently expressed in human $\mathrm{T}$ cells without impairing pheno-
\end{abstract}

type, function, or antigen specificity. This reagent is based on a modified human caspase 9 fused to a human FK506 binding protein (FKBP) to allow conditional dimerization using a small molecule pharmaceutical. A single 10-nM dose of synthetic dimerizer drug induces apoptosis in $99 \%$ of transduced cells selected for high transgene expression in vitro and in vivo. This system has several advantages over currently available suicide genes. First, it consists of human gene products with low potential immunogenicity. Second, administration of dimerizer drug has no effects other than the selective elimination of transduced $T$ cells. Third, inducible caspase 9 maintains function in $\mathrm{T}$ cells overexpressing antiapoptotic molecules. These characteristics favor incorporation of inducible caspase 9 as a safety feature in human T-cell therapies. (Blood. 2005;105:4247-4254)

(c) 2005 by The American Society of Hematology

\section{Introduction}

Cellular therapies hold great promise for the treatment of human disease, and this promise may be extended if the cells are first genetically modified to alter or augment function. Unfortunately, significant toxicities from the cells themselves or from their transgene products have hampered clinical investigation. There is considerable interest in developing means by which infused cells may be ablated should problems arise from their use. Most experience with safety-switch genes to date has been in $\mathrm{T}$ lymphocytes since immunotherapy with these cells has proved efficacious as treatment for viral infections and malignancies. ${ }^{1-4}$

The herpes simplex virus I-derived thymidine kinase (HSVTK) gene has been used as an in vivo suicide switch in donor T-cell infusions to treat recurrent malignancy and Epstein Barr virus (EBV) lymphoproliferation after hemopoietic stem cell transplantation. ${ }^{5,6}$ However, destruction of $\mathrm{T}$ cells causing graft-versus-host disease was incomplete, and the use of ganciclovir (or analogs) as a pro-drug to activate HSV-TK precludes administration of ganciclovir as an antiviral drug for cytomegalovirus infections. Moreover, HSV-TK-directed immune responses have resulted in elimination of HSV-TK-transduced cells, even in immunosuppressed human immunodeficiency virus and bone marrow transplant patients, compromising the persistence and hence efficacy of the infused $\mathrm{T}$ cells. ${ }^{5,7}$ The $E$ coli-derived cytosine deaminase gene has also been used clinically, ${ }^{8}$ but as a xenoantigen it is also likely to be immunogenic and thus incompatible with T-cell-based therapies that require long-term persistence.

From the Center for Cell and Gene Therapy; the Departments of Pediatrics, Medicine, Immunology, and Molecular Virology and Microbiology, Baylor College of Medicine; the Methodist Hospital; and Texas Children's Hospital, Houston, TX.

Submitted December 1, 2004; accepted January 31, 2005. Prepublished online as Blood First Edition Paper, February 22, 2005; DOI 10.1182/blood-2004-11-4564.

Supported in part by grants from The Dutch Cancer Foundation, The Royal Dutch Academy for Science, The Ank van Vlissingen Foundation (K.C.S.); The British Society for Haematology Society Fellowship (M.A.P.); DOD no. PC020 062 (D.M.S.), NCI PO1 CA94 237, a Specialized Centre of Research Award from the Leukemia Lymphoma Society, a Translational Research Award
Transgenic human CD20, which can be activated by a monoclonal chimeric anti-CD20 antibody, has been proposed as a nonimmunogenic safety system. ${ }^{9}$ However, it results in the unwanted loss of normal B cells for 6 months or more. An alternative suicide gene strategy is based on human proapoptotic molecules fused with an FKBP variant that is optimized to bind a chemical inducer of dimerization (CID), ${ }^{10}$ AP1903, a synthetic drug that has proven safe in healthy volunteers. ${ }^{11}$ Administration of this small molecule results in cross-linking and activation of the proapoptotic target molecules. The application of this inducible system in human $\mathrm{T}$ lymphocytes has been explored using Fas or the death effector domain (DED) of the Fas-associated death domain-containing protein (FADD) as proapoptotic molecules. Up to $90 \%$ of $\mathrm{T}$ cells transduced with these inducible death molecules underwent apoptosis after administration of CID. ${ }^{12-16}$

While these results are promising, elimination of $90 \%$ of transduced cells may be insufficient to ensure safety of genetically modified cells in vivo. Moreover, death molecules that act downstream of most apoptosis inhibitors may be effective in a wider range of cells. The activity of membrane proximal apoptosis initiators such as Fas and FADD may be impaired when cellular inhibitors of apoptosis such as c-FLIP, bcl-2, and bcl- $\mathrm{x}_{\mathrm{L}}$ are upregulated (Figure 1) - a frequent early event in malignant transformation and in the long-term maintenance of memory T cells. ${ }^{17,18}$ Hence, the most deleterious cells may be inadvertently spared.

from the Leukemia Lymphoma Society (C.M.R.), and a Doris Duke Distinguished Clinical Scientist Award (H.E.H.).

The online version of this article contains a data supplement.

Reprints: Cliona M. Rooney, Center for Cell and Gene Therapy, 1102 Bates St, Suite 750.09, Houston, TX 77030; e-mail: crooney@bcm.tmc.edu.

The publication costs of this article were defrayed in part by page charge payment. Therefore, and solely to indicate this fact, this article is hereby marked "advertisement" in accordance with 18 U.S.C. section 1734.

C 2005 by The American Society of Hematology 


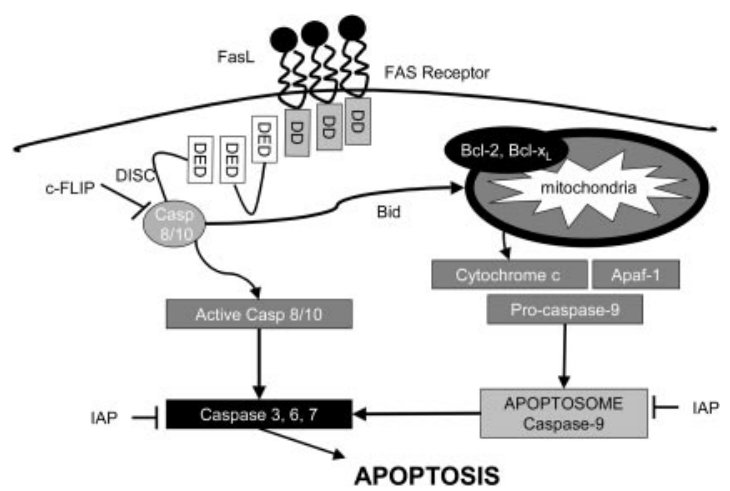

Figure 1. Antiapoptotic molecules regulate the sensitivity to apoptotic signals. In human lymphocytes apoptosis can be induced through at least 2 pathways. Stimulation of the Fas receptor results in recruitment of the initiator caspase 8 , through interaction with the adaptor molecule Fas-associated death domain protein (FADD) by means of its death domains (DDs) and death effector domains (DEDs). In turn, activated caspase 8 activates the effector caspases 3, 6, and 7. Alternatively, disruption of the mitochondrial membrane results in the release of cytochrome $c$, which activates caspase 9 through interaction with the adaptor molecule, apoptotic protease-activating factor 1 (Apaf-1). Caspase 9 is then able to activate caspase 3 . The death receptor-activated extrinsic pathway can crosstalk to the intrinsic mitochondrial pathway through the caspase 8-mediated cleavage of Bid (BH3 interacting domain death agonist). Inhibitors of apoptosis that engage at different steps of these pathways regulate the balance between apoptosis and survival. FLIP prevents the full activation of caspase 8 . Antiapoptotic bcl-2 (B-cell CLL/lymphoma-2) family members prevent apoptosis initiated via the mitochondria. Inhibitors of apoptosis proteins (IAPs including X-linked IAP [XIAP]) can either prevent activation of caspases 3,7 , and 9 or inhibit their activated forms. DISC indicates death-inducing signaling complex; FasL, Fas ligand; c-FLIP, cellular FLICE-like inhibitory protein.

The efficacy of adoptive immunotherapy may be enhanced by rendering the therapeutic $\mathrm{T}$ cells resistant to immune evasion strategies employed by tumor cells. In vitro studies have shown that this can be achieved by transduction with a dominant-negative receptor or an immunomodulatory cytokine. ${ }^{19,20}$ Moreover, transfer of antigen-specific T-cell receptors allows for the application of T-cell therapy to a broader range of tumors. ${ }^{21,22}$ We therefore chose to develop and test a suicide system for engineered human $\mathrm{T}$ cells to allow their subsequent use in clinical studies. Here we describe how a modification of a late-stage apoptosis pathway molecule, caspase 9, can be stably expressed in human $\mathrm{T}$ lymphocytes without compromising their functional and phenotypic characteristics while demonstrating exquisite sensitivity to CID, even in $\mathrm{T}$ cells that have upregulated antiapoptotic molecules.

\section{Materials and methods}

\section{Plasmids}

Full-length inducible caspase 9 (F'F-C-Casp9.I.GFP) consists of full-length caspase 9, including its caspase recruitment domain (CARD; GenBank NM001 229) linked to 2 12-kDa human FK506 binding proteins (FKBP12; GenBank AH002 818) that contain an F36V mutation (Figure 2A). ${ }^{10}$ The inducer of dimerization used in this study, AP20187 (generous gift of ARIAD Pharmaceuticals, Cambridge, MA), is a nontoxic synthetic FK506 analog that has been modified to reduce interactions with endogenous FKBPs, while enhancing binding to this FK506-BP12 variant. Administration of the CID results in the aggregation of inducible caspase 9 molecules, leading to their activation. Caspase 9 will subsequently activate downstream effector caspases, such as caspase 3 , and ultimately induce apoptosis (Figure 1). Silent mutations in the third base of multiple codons have been introduced into the first FKBP segment to prevent homologous recombination between the coding sequences of the 2 linked FKBPs in our retroviral system $^{12}$ and a short Ser-Gly-Gly-Gly-Ser linker connects the FKBPs and caspase 9 to enhance flexibility. ${ }^{14}$ Inducible Fas consists of the extracellular and transmembrane domains of human low-affinity nerve growth factor receptor ( $\triangle$ NGFR), 2 FKBP $12_{\mathrm{V} 36} \mathrm{~s}$, and the cytoplasmic domains of human Fas as described by Thomis et al. ${ }^{12}$ The cDNA for the p40 and p35 subunit of human interleukin 12 (IL-12) connected with a flexible linker was cloned from pcDNA3.1, a kind gift from Robert Anderson and Grant Prentice, Royal Free Hospital, London, United Kingdom. ${ }^{23}$ All constructs were cloned into the retroviral vector MSCV.IRES.GFP.

\section{Western blot}

Transfected 293T cells were resuspended in lysis buffer (50\% Tris/Gly, $10 \%$ sodium dodecyl sulfate [SDS], $4 \%$ beta-mercaptoethanol, $10 \%$ glycerol, $12 \%$ water, $4 \%$ bromophenol blue at $0.5 \%$ ) containing aprotinin, leupeptin, and phenylmethylsulfonyl fluoride (Boehringer, Ingelheim, Germany) and incubated for 30 minutes on ice. After a 30-minute centrifugation, supernatant was harvested, mixed 1:2 with Laemmli buffer (Bio-Rad, Hercules, CA), boiled and loaded on a 10\% SDS-polyacrylamide gel. The membrane was probed with rabbit anti-caspase 9 (amino acid residues 299-318) immunoglobulin G (IgG; Affinity BioReagents, Golden, CO; 1:500) and with mouse anti-GFP IgG (Covance, Berkeley, CA; 1:25 000). Blots were then exposed to appropriate peroxidase-coupled secondary antibodies and protein expression was detected with enhanced chemiluminescence (ECL; Amersham, Arlington Heights, IL). The membrane was

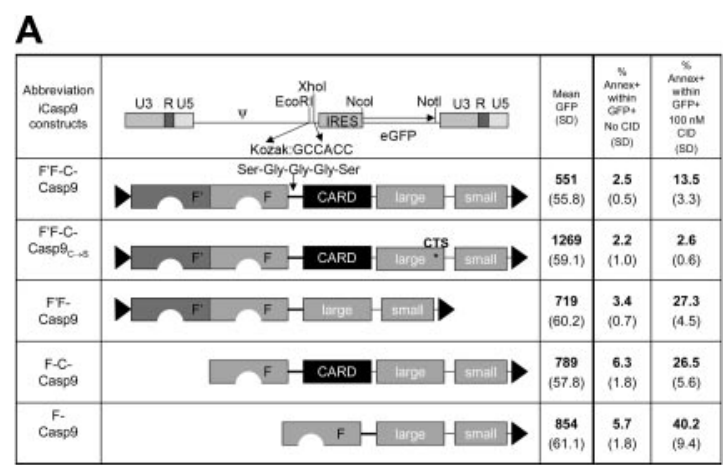

B

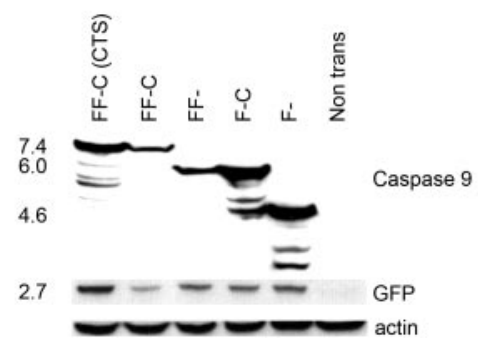

Figure 2. Modifications of full-length inducible caspase 9. (A) The full-length inducible caspase 9 molecule (F'-F-C-Casp9) consists of 2 FK506 binding proteins (FKBPs) linked with a Ser-Gly-Gly-Gly-Ser linker to the small and large subunit of the caspase molecule. The amino acid sequence of one of the FKBPs (F') is codonwobbled to prevent homologous recombination when expressed in a retrovirus. F'F-C-Casp $9_{\mathrm{C} \rightarrow \mathrm{S}}$ contains a cysteine to serine mutation at position 287 that disrupts its activation site. In constructs F'F-Casp9, F-C-Casp9, and F'-Casp9, either the caspase activation domain (CARD), one FKBP, or both, were deleted respectively. All constructs were cloned into MSCV.IRES.GFP as EcoRI-Xhol fragments. 293T cells were transfected with each of these constructs and 48 hours after transduction expression of the marker gene GFP was analyzed by flow cytometry. In addition, 24 hours after transfection, 293T cells were incubated overnight with $100 \mathrm{nM} \mathrm{CID}$ and subsequently stained with the apoptosis marker annexin $\mathrm{V}$. The mean and standard deviation of transgene expression level (mean GFP) and number of apoptotic cells before and after exposure to CID (\% annexin $\mathrm{V}$ within $\mathrm{GFP}^{+}$cells) from 4 separate experiments are shown. (B) Coexpression of the inducible caspase 9 constructs of the expected size with the marker gene GFP in transfected 293T cells was demonstrated by Western blot using a caspase 9 antibody specific for amino acid residues 299-318, present both in the full-length and truncated caspase molecules as well as a GFP-specific antibody. Additional smaller size bands likely represent degradation products. Degradation products for the F'F-C-Casp9 and F'F-Casp9 constructs may not be detected due to a lower expression level of these constructs as a result of their basal activity. Equal loading was confirmed by blotting for actin. 
then stripped and reprobed with goat polyclonal antiactin (Santa Cruz Biotechnology; 1:500) to check equality of loading.

\section{Cell lines}

B95-8 EBV transformed B-cell lines (LCLs), Jurkat, and MT-2 cells (kindly provided by Dr S. Marriott, Baylor College of Medicine, Houston, TX) were cultured in RPMI 1640 (Hyclone, Logan, UT) containing 10\% fetal bovine serum (FBS; Hyclone). Polyclonal EBV-specific T-cell lines were cultured in 45\% RPMI/45\% Clicks (Irvine Scientific, Santa Ana, CA) $/ 10 \%$ FBS and generated as previously reported. ${ }^{2}$ Briefly, peripheral blood mononuclear cells $\left(2 \times 10^{6}\right.$ per well of a 24 -well plate $)$ were stimulated with autologous LCLs irradiated at 4000 rads at a responder-to-stimulator $(\mathrm{R} / \mathrm{S})$ ratio of 40:1. After 9 to 12 days, viable cells were restimulated with irradiated LCLs at an R/S ratio of $4: 1$. Subsequently, cytotoxic $\mathrm{T}$ cells (CTLs) were expanded by weekly restimulation with LCLs in the presence of $40 \mathrm{U} / \mathrm{mL}$ to $100 \mathrm{U} / \mathrm{mL}$ recombinant human interleukin-2 (rhIL-2; Proleukin; Chiron, Emeryville, CA).

\section{Retrovirus transduction}

For the transient production of retrovirus, 293T cells were transfected with iCasp9/iFas constructs, along with plasmids encoding gag-pol and RD114 envelope using GeneJuice transfection reagent (Novagen, Madison, WI). Virus was harvested 48 to 72 hours after transfection, snap frozen, and stored at $-80^{\circ} \mathrm{C}$ until use. A stable FLYRD18-derived retroviral producer line was generated by multiple transductions with VSV-G pseudotyped transient retroviral supernatant. ${ }^{24}$ FLYRD18 cells with highest transgene expression were single-cell sorted, and the clone that produced the highest virus titer was expanded and used to produce virus for lymphocyte transduction. The transgene expression, function, and retroviral titer of this clone was maintained during continuous culture for more than 8 weeks. For transduction of human lymphocytes, a non-tissue-culture-treated 24-well plate (Becton Dickinson, San Jose, CA) was coated with recombinant fibronectin fragment (FN CH-296; Retronectin; Takara Shuzo, Otsu, Japan; $4 \mu \mathrm{g} / \mathrm{mL}$ in PBS, overnight at $4^{\circ} \mathrm{C}$ ) and incubated twice with $0.5 \mathrm{~mL}$ retrovirus per well for 30 minutes at $37^{\circ} \mathrm{C}$. Subsequently, $3 \times 10^{5}$ to $5 \times 10^{5} \mathrm{~T}$ cells per well were transduced for 48 to 72 hours using $1 \mathrm{~mL}$ virus per well in the presence of $100 \mathrm{U} / \mathrm{mL}$ IL-2. Transduction efficiency was determined by analysis of expression of the coexpressed marker gene green fluorescent protein (GFP) on a FACScan flow cytometer (Becton Dickinson). For functional studies, transduced CTLs were either nonselected or segregated into populations with low, intermediate, or high GFP expression using a MoFlo cytometer (Dako Cytomation, Ft Collins, CO) as indicated.

\section{Induction and analysis of apoptosis}

CID (AP20187; ARIAD Pharmaceuticals) at indicated concentrations was added to transfected $293 \mathrm{~T}$ cells or transduced CTLs. Adherent and nonadherent cells were harvested and washed with annexin binding buffer (BD Pharmingen, San Jose, CA). Cells were stained with annexin-V and 7-amino-actinomycin D (7-AAD) for 15 minutes according to the manufacturer's instructions (BD Pharmingen). Within 1 hour after staining, cells were analyzed by flow cytometry using CellQuest software (Becton Dickinson).

\section{Cytotoxicity assay}

The cytotoxic activity of each CTL line was evaluated in a standard 4-hour ${ }^{51} \mathrm{Cr}$ release assay, as previously described. ${ }^{25}$ Target cells included autologous LCLs, human leukocyte antigen (HLA) class I-mismatched LCLs and the lymphokine-activated killer cell-sensitive T-cell lymphoma line HSB-2. Target cells incubated in complete medium or 1\% Triton X-100 (Sigma, St Louis, MO) were used to determine spontaneous and maximum ${ }^{51} \mathrm{Cr}$ release, respectively. The mean percentage of specific lysis of triplicate wells was calculated as $100 \times$ (experimental release - spontaneous release) / (maximal release - spontaneous release).

\section{Phenotyping}

Cell-surface phenotype was investigated using the following monoclonal antibodies: CD3, CD4, CD8, (Becton Dickinson) and CD56 and TCR- $\alpha / \beta$ (Immunotech, Miami, FL). $\triangle$ NGFR-iFas was detected using anti-NGFR antibody (Chromaprobe, Aptos, CA). Appropriate matched isotype controls (Becton Dickinson) were used in each experiment. Cells were analyzed with a FACSscan flow cytometer (Becton Dickinson).

\section{Analysis of cytokine production}

The concentration of interferon- $\gamma$ (IFN- $\gamma$ ), IL-2, IL-4, IL-5, IL-10, and tumor necrosis factor- $\alpha(\mathrm{TNF} \alpha)$ in CTL culture supernatants was measured using the Human Th1/Th2 cytokine cytometric Bead Array (BD Pharmingen) and the concentration of IL-12 in the culture supernatants was measured by enzyme-linked immunosorbent assay (ELISA; R\&D Systems, Minneapolis, MN) according to the instructions of the manufacturer.

\section{In vivo experiments}

Nonobese diabetic severe combined immunodeficient (NOD/SCID) mice, 6 to 8 weeks of age, were irradiated $(250 \mathrm{rad})$ and injected subcutaneously in the right flank with $10 \times 10^{6}$ to $15 \times 10^{6}$ LCLs resuspended in Matrigel (BD Bioscience). Two weeks later mice bearing tumors that were approximately $0.5 \mathrm{~cm}$ in diameter were injected into the tail vein with a $1: 1$ mixture

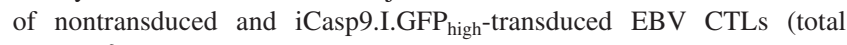
$15 \times 10^{6}$ ). At 4 to 6 hours prior and 3 days after CTL infusion, mice were injected intraperitoneally with recombinant hIL-2 (2000 U; Proleukin; Chiron). On day 4, the mice were randomly segregated in 2 groups: 1 group received CID (50 $\mu \mathrm{g}$ AP20 187, intraperitoneally) and 1 group received carrier only (16.7\% propanediol, $22.5 \%$ PEG400, and $1.25 \%$ Tween 80 , intraperitoneally). On day 7, all mice were killed. Tumors were homogenized and stained with antihuman CD3 (BD Pharmingen). By FACS analysis, the number of $\mathrm{GFP}^{+}$cells within the gated $\mathrm{CD}^{+}$population was evaluated. Tumors from a control group of mice that received only nontransduced CTLs (total $15 \times 10^{6}$ ) were used as a negative control in the analysis of $\mathrm{CD}^{+} / \mathrm{GFP}^{+}$cells.

\section{Results}

Optimization of expression and function of inducible caspase 9

We initially screened previously described caspases 3, 7, and 9 for their suitability as inducible safety-switch molecules both in transfected 293T cells and in transduced human T cells. ${ }^{14}$ Only inducible caspase 9 (iCasp9) could be expressed at levels sufficient to confer sensitivity to CID (data not shown). However, even the initially tested iCasp9 could not be maintained stably at high levels in T cells, and we hypothesized that basal activity of the transgene was eliminating transduced cells. The CARD domain is responsible for physiologic dimerization of caspase 9 molecules, by a cytochrome $\mathrm{C}$ and adenosine triphosphate (ATP)-driven interaction with apoptotic protease-activating factor 1 (Apaf-1). Hence the CARD domain appears superfluous in this context and its removal might reduce basal activity. Given that only dimerization rather than multimerization is required for activation of caspase 9 , we also reasoned that a single FKBP domain might be adequate to effect activation. We therefore compared the activity of iCasp9 derivatives in which either the CARD domain, or one of the 2 FKBP domains, or both, had been removed. A construct with a disrupted activation site, F' F-C-Casp9 ${ }_{\mathrm{C} \rightarrow \mathrm{S}}$, provided a nonfunctional control (Figure 2A). All constructs were cloned into the retroviral vector $\mathrm{MSCV}^{26}$ in which retroviral long terminal repeats (LTRs) direct transgene expression and enhanced GFP is coexpressed from the same mRNA by use of an internal ribosomal entry site (IRES). In transfected $293 \mathrm{~T}$ cells, expression of all inducible caspase 9 
constructs at the expected size as well as coexpression of GFP was demonstrated by Western blot (Figure 2B). Protein expression (estimated by mean fluorescence of GFP and visualized on Western blot) was highest in the nonfunctional construct F' F-C-Casp $9_{\mathrm{C} \rightarrow \mathrm{S}}$ and greatly diminished in the full-length construct F'F-C-Casp9. Removal of the CARD (F'F-Casp9), one FKBP (F-C-Casp9), or both (F-Casp9) resulted in progressively higher expression of both inducible caspase 9 and GFP, and correspondingly enhanced sensitivity to CID (Figure 2A). Based on these results, the F-Casp9 construct (henceforth referred to as iCasp9 $9_{M}$ ) was used for further study in human T lymphocytes.

\section{Stable expression of iCasp $9_{M}$ in human T lymphocytes}

The long-term stability of suicide gene expression is of utmost importance, since suicide genes must be expressed for as long as the genetically engineered cells persist. For T-cell transduction, a FLYRD18-derived retroviral producer clone that produces hightiter RD114-pseudotyped virus was generated to facilitate the transduction of $\mathrm{T}$ cells. ${ }^{24}$ We evaluated iCasp $9_{\mathrm{M}}$ expression in EBV-specific CTL lines (EBV-CTL), since these have wellcharacterized function and specificity and are already being used as in vivo therapy for prevention and treatment of EBV-associated malignancies. ${ }^{2,27}$ Consistent transduction efficiencies of EBVCTLs of more than $70 \%$ (mean, $75.3 \%$; range, $71.4 \%-83.0 \%$ in 5 different donors) were obtained after a single transduction with retrovirus. The expression of $\mathrm{iCasp}_{\mathrm{M}}$ in EBV-CTLs was stable for at least 4 weeks after transduction without selection or loss of transgene function (data not shown).

\section{iCasp $9_{\mathrm{M}}$ does not alter transduced T-cell characteristics}

To ensure that expression of iCasp $9_{M}$ did not alter T-cell characteristics, we compared the phenotype, antigen-specificity, proliferative potential, and function of nontransduced or nonfunctional iCasp $9_{\mathrm{C} \rightarrow \mathrm{S}}$-transduced EBV-CTLs, with that of $\mathrm{iCasp} 9_{\mathrm{M}^{-}}$-transduced EBV-CTLs. In 4 separate donors, transduced and nontransduced CTLs consisted of equal numbers of $\mathrm{CD} 4^{+}, \mathrm{CD} 8^{+}, \mathrm{CD}^{+} 6^{+}$, and TCR $\alpha \beta^{+}$cells (Figure 3A). Similarly, production of cytokines including IFN- $\gamma$, TNF $\alpha$, IL-10, IL-4, IL-5, and IL-2 was unaltered by iCasp $9_{M}$ expression (Figure 3B). iCasp $9_{M}$-transduced EBVCTLs specifically lysed autologous LCLs comparable to nontransduced and control-transduced CTLs (Figure 3C). Expression of iCasp9 $9_{M}$ did not affect the growth characteristics of exponentially growing CTLs, and importantly, dependence on antigen and IL-2 for proliferation was preserved (Figure 3D).

\section{Elimination of more than $99 \%$ of T lymphocytes selected for high transgene expression in vitro}

To provide an effective safety switch, suicide gene induction should eliminate all gene-modified cells. Therefore, iCasp $9_{M}$ proficiency in CTLs was tested by monitoring loss of GFPexpressing cells after administration of CID; $91.3 \%$ (range, $89.5 \%$ $92.6 \%$ in 5 different donors) of $\mathrm{GFP}^{+}$cells were eliminated after a single 10-nM dose of CID (Figure 4A). Similar results were obtained regardless of exposure time to CID (range, 1 hourcontinuous; data not shown). In all experiments, CTLs that survived CID treatment had low transgene expression with a $70 \%$ (range, 55\%-82\%) reduction in mean fluorescence intensity of GFP after CID. No further elimination of the surviving $\mathrm{GFP}^{+} \mathrm{T}$ cells could be obtained by an antigenic stimulation followed by a second 10-nM dose of CID (data not shown). Therefore, the nonresponding CTLs most likely expressed insufficient $\mathrm{iCasp} 9_{\mathrm{M}}$ for functional
A

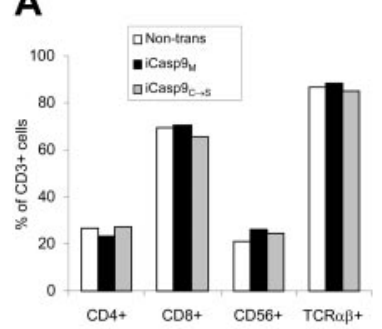

$\mathrm{C}_{70}$

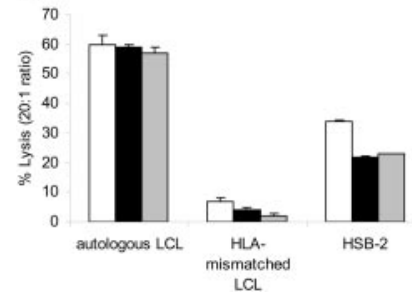

Figure 3. Expression of iCasp $9_{M}$ does not affect the phenotype or function of EBV-CTLs. Phenotype (A) and secretion (B) of Th1- and Th2-type cytokines upon antigen stimulation, and $(C)$ cytolytic activity against autologous EBV-transformed lymphoblastoid B-cell line (LCL), HLA-mismatched LCL, and HSB-2 (a LAK cell target) were compared in nontransduced $(\square)$, F-Casp $9_{\mathrm{M}}$-transduced $(\square)$, and F'F-C-Casp9 $9_{\mathrm{C} \rightarrow \mathrm{S}}$-transduced (圆) EBV-specific CTLs (EBV-CTLs) on day 15 to day 18 after transduction (2 antigenic stimulations after transduction). The mean and standard deviation of triplicate wells are shown. Examples of experiments using EBV-CTLs from 4 different donors are shown. ${ }^{*}$ Greater than $5000 \mathrm{pg} / \mathrm{mL}$. (D) On day 21 after transduction the normal weekly antigenic stimulation with autologous LCLs and IL-2 was continued ( $\bullet$ ) or discontinued $(\square)$ to evaluate the antigen dependence of iCasp $9_{\mathrm{M}}$-transduced CTLs.

activation by CID. To test this hypothesis CTLs were sorted for low, intermediate, and high expression of the linked marker gene GFP and mixed 1:1 with nontransduced CTLs from the same donor to allow for an accurate quantitation of the number of transduced $\mathrm{T}$ cells responding to CID-induced apoptosis. The number of transduced T cells eliminated increased with the level of GFP transgene expression (Figure S1; see the Supplemental Figure link at the top of the online article on the Blood website). For $\mathrm{GFP}_{\text {high }}$-selected cells, $10 \mathrm{nM}$ CID led to deletion of $99.1 \%$ (range, $98.7 \%-99.4 \%$ ) of transduced cells (Figure 4A). Rapid induction of apoptosis in these $\mathrm{GFP}_{\text {high-selected cells is demonstrated by apoptotic characteristics }}$ such as cell shrinkage and fragmentation within 14 hours of CID administration (Figure 4B). Of these T cells, 64\% (range, 59\%$69 \%$ ) had an apoptotic (annexin- $\mathrm{V}^{+} / 7-\mathrm{AAD}^{-}$) and $30 \%$ (range, $26 \%-32 \%$ ) had a necrotic (annexin- $\mathrm{V}^{+} / 7-\mathrm{AAD}^{+}$) phenotype (Figure $4 \mathrm{C}$ ). In contrast, the induction of apoptosis was significantly lower in $\mathrm{T}$ cells selected for intermediate or low GFP expression (Figure S1). For clinical applications therefore, transduced cells may have to be sorted for sufficient transgene expression before administration. CID-induced apoptosis was inhibited by the pancaspase inhibitor zVAD-fmk (100 $\mu \mathrm{M}$ for 1 hour prior to adding CID; data not shown). Titration of CID showed that $1 \mathrm{nM}$ CID was sufficient to obtain the maximal deletion effect (Figure 4D). This dose response remained unchanged for at least 4 weeks after transduction (data not shown).

\section{iCasp $9_{\mathrm{M}}$ is functional in malignant cells that express antiapoptotic molecules}

We had selected caspase 9 as an inducible proapoptotic molecule for clinical use rather than previously described iFas ${ }^{12}$ and iFADD, ${ }^{16}$ since caspase 9 acts relatively late in apoptosis signaling and should be less susceptible to inhibition by apoptosis inhibitors. Thus, suicide function should be preserved not only in malignant, 
From www.bloodjournal.org at UCL Library Services on September 16, 2008. For personal use only.

B
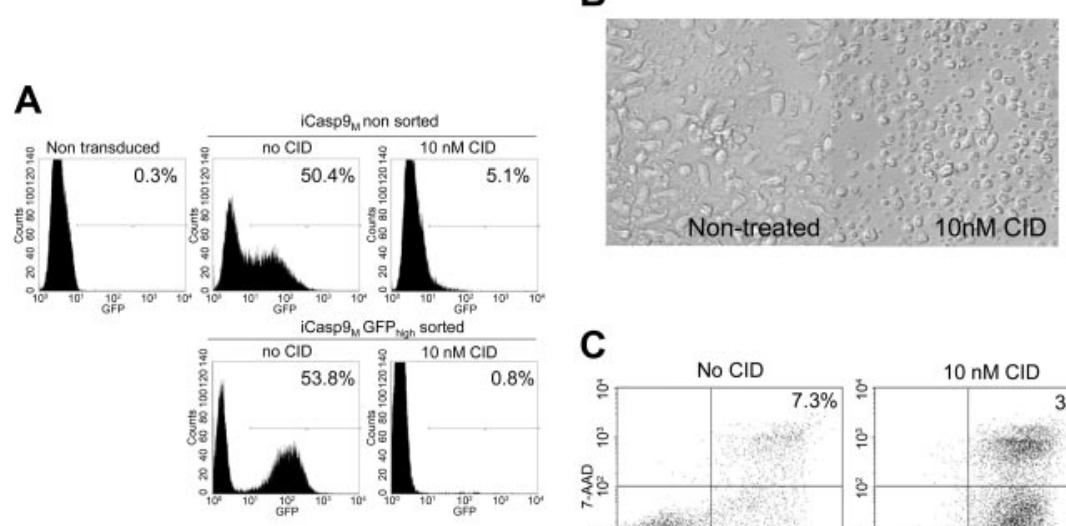

D
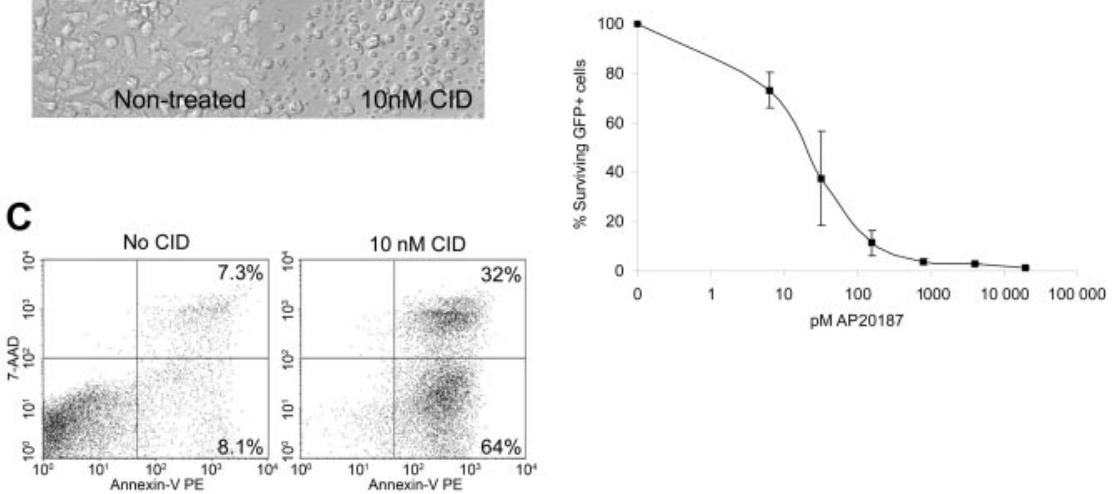

Figure 4. Administration of CID eliminates iCasp $9_{\mathrm{M}}$-expressing T cells. (A) On the day of antigen stimulation, F-Casp $9_{\mathrm{M}}$.I.GFP-transduced CTLs were either untreated or treated with $10 \mathrm{nM}$ CID. Seven days later, the response to CID was measured by flow cytometry for GFP. The percentage of transduced T cells was adjusted to $50 \%$ to allow for

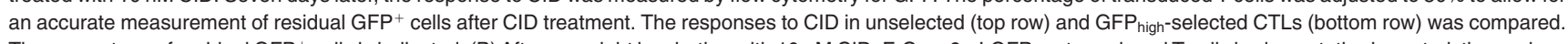
The percentage of residual GFP ${ }^{+}$cells is indicated. (B) After overnight incubation with $10 \mathrm{nM} \mathrm{CID,} \mathrm{F-Casp} 9_{\mathrm{M}}$ I.GFP $_{\text {high-transduced T cells had apoptotic characteristics such as }}$ cell shrinkage and fragmentation by microscopic evaluation. (C) Staining with markers of apoptosis showed that $64 \%$ of $\mathrm{T}$ cells had an apoptotic phenotype (annexin $\mathrm{V}^{+}$, 7-AAD ${ }^{-}$, lower right quadrant) and $32 \%$ a necrotic phenotype (annexin $\mathrm{V}^{+}, 7-\mathrm{AAD}^{+}$, upper right quadrant). A representative example of 3 separate experiments is shown. (D) $\mathrm{A}$ dose-response curve using the indicated amounts of CID (AP20187) shows the sensitivity of F-Casp9 $9_{\mathrm{M}}$. I.GFP high to CID. Survival of GFP ${ }^{+}$cells is measured on day 7 after administration of the indicated amount of CID. Shown are mean and standard deviation. Similar results were obtained using AP1903, which has proven safe in a clinical trial in healthy volunteers. ${ }^{11}$

transformed T-cell lines that express antiapoptotic molecules, ${ }^{28,29}$ but also in subpopulations of normal $\mathrm{T}$ cells that express elevated antiapoptotic molecules as part of the process to ensure long-term preservation of memory cells. ${ }^{18,30}$ To test this hypothesis we first compared the function of $i$ Casp $9_{M}$ and iFas in EBV-CTLs. Like iCasp9, inducible Fas ${ }^{12}$ was expressed by the MSCV.IRES.GFP vector. For these experiments both $\triangle$ NGFR.iFas.I.GFP and iCasp $9_{\mathrm{M}}$.I.GFP-transduced CTLs were sorted for $\mathrm{GFP}_{\text {high }}$ expression and mixed with nontransduced CTLs at a 1:1 ratio to obtain cell populations that expressed either iFas or iCasp9 $9_{M}$ at equal proportions and at similar levels (Figure 5A). Elimination of $\mathrm{GFP}^{+}$cells after administration of $10 \mathrm{nM}$ CID was more rapid and more efficient in iCasp $_{M}$ than in iFas-transduced CTLs $\left(99.2 \% \pm 0.14 \%\right.$ of iCasp $_{\mathrm{M}}$-transduced cells compared with $89.3 \% \pm 4.9 \%$ of iFas-transduced cells at day 7 after CID; $P<.05$; Figure 5B).

Second, we compared the function of iCasp9 $9_{M}$ and iFas in 2 malignant T-cell lines: Jurkat, an apoptosis-sensitive T-cell leukemia line, and MT-2, an apoptosis-resistant T-cell line, due to c-FLIP and bcl- $\mathrm{x}_{\mathrm{L}}$ expression. ${ }^{31,32}$ Jurkat cells and MT-2 cells were transduced with iFas and iCasp $9_{M}$ with similar efficiencies (92\% vs $84 \%$ in Jurkat, $76 \%$ vs $70 \%$ in MT-2) and were cultured in the presence of $10 \mathrm{nM}$ CID for 8 hours. Annexin-V staining showed that although iFas and iCasp $9_{\mathrm{M}}$ induced apoptosis in an equivalent number of Jurkat cells $(56.4 \% \pm 15.6 \%$ and $57.2 \% \pm 18.9 \%$, respectively), only activation of iCasp $9_{\mathrm{M}}$ resulted in apoptosis of MT-2 cells $(19.3 \% \pm 8.4 \%$ and $57.9 \% \pm 11.9 \%$ for iFas and iCasp $9_{M}$, respectively; Figure $5 \mathrm{C}$ ). These results demonstrate that in $\mathrm{T}$ cells overexpressing apoptosis-inhibiting molecules, the function of iFas can be blocked, while iCasp $9_{M}$ can still effectively induce apoptosis.

\section{iCasp $9_{\mathrm{M}}$-mediated elimination of $\mathrm{T}$ cells expressing an immunomodulatory transgene}

To determine whether iCasp $9_{M}$ could effectively destroy cells genetically modified to express an active transgene product, we measured the ability of iCasp $9_{M}$ to eliminate EBV-CTLs stably expressing IL-12 to enhance their antitumor activity. ${ }^{20}$ While IL-12 was undetectable in the supernatant of nontransduced and iCasp9 $9_{M}$.IRES.GFP-transduced CTLs, the supernatant of iCasp $9_{\mathrm{M}}$.IRES.IL-12-transduced cells contained $324 \mathrm{pg} / \mathrm{mL}$ to

A

B
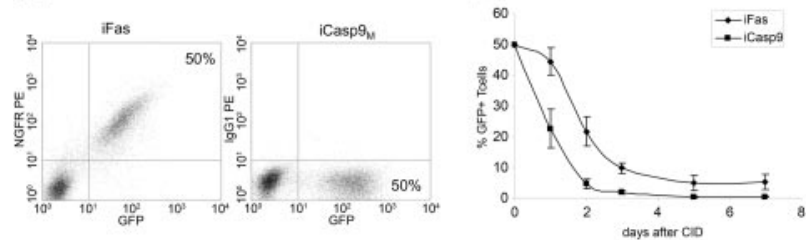

C

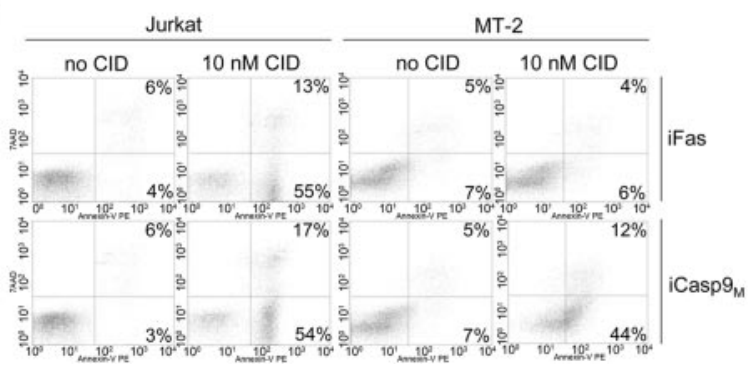

Figure 5. Comparison of functionality of iFas and iCasp $9_{M}$ in $T$ cells. (A) EBV-CTLs were transduced with $\Delta$ NGFR-iFas.I.GFP (left) or iCasp $9_{M}$.I.GFP (right) and sorted for high GFP expression. Transduced CTLs were then mixed 1:1 with nontransduced CTLs. The percentages of $\Delta \mathrm{NGFR}^{+} / \mathrm{GFP}^{+}$and $\mathrm{GFP}^{+} \mathrm{T}$ cells are indicated. (B) On the day of LCL stimulation, $10 \mathrm{nM}$ CID was administered, and GFP was measured at the time points indicated to determine the response to CID.

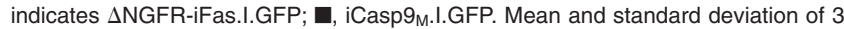
experiments are shown. (C) The human T-cell lines Jurkat (left) and MT-2 (right) were

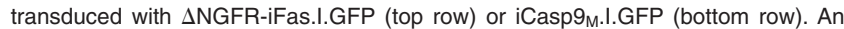
equal percentage of $\mathrm{T}$ cells was transduced with each of the suicide genes: $92 \%$ for $\Delta$ NGFR-iFas.I.GFP versus $84 \%$ for iCasp $9_{\mathrm{M}}$.I.GFP in Jurkat, and $76 \%$ for $\Delta$ NGFRiFas.I.GFP versus $70 \%$ for iCasp9 $9_{M}$.I.GFP in MT-2 (data not shown). T cells were either nontreated or incubated with $10 \mathrm{nM}$ CID. Eight hours after exposure to CID, apoptosis was measured by staining for annexin $V$ and 7-AAD. Representative example of 3 experiments is shown. PE indicates phycoerythrin. 


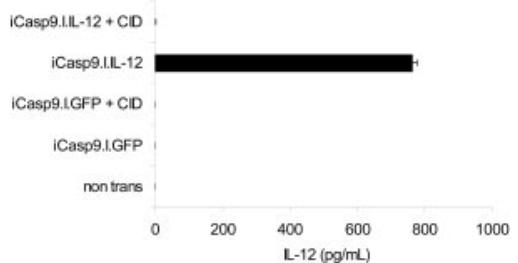

Figure 6. Function of iCasp $9_{M}$ when coexpressed with IL-12. The marker gene GFP in the iCasp $9_{M}$.I.GFP constructs was replaced by flexi IL-12, encoding the p40 and p35 subunits of human IL-12. iCasp $9_{\mathrm{M}}$.I.GFP- and iCasp $9_{\mathrm{M}}$.I.IL-12-transduced EBV-CTLs were stimulated with LCLs, and then left untreated or exposed to $10 \mathrm{nM}$ CID. Three days after a second antigenic stimulation, IL-12 in the culture supernatant was measured by IL-12 ELISA (detection limit of this assay is $7.8 \mathrm{pg} / \mathrm{mL}$ ). The mean and standard deviation of triplicate wells are indicated. Results of 1 of 2 experiments with CTLs from 2 different donors are shown.

$762 \mathrm{pg} / \mathrm{mL}$ IL-12. After administration of $10 \mathrm{nM} \mathrm{CID}$, however, the IL-12 in the supernatant fell to undetectable levels $(<7.8$ $\mathrm{pg} / \mathrm{mL}$ ). Hence, even without prior sorting for high transgeneexpressing cells, activation of iCasp $9_{M}$ is sufficient to completely eliminate all $\mathrm{T}$ cells producing biologically relevant levels of IL-12 (Figure 6).

\section{Elimination of more than $99 \%$ of T cells selected for high transgene expression in vivo}

Finally, to evaluate the function of $\mathrm{iCasp} 9_{\mathrm{M}}$ in transduced EBVCTLs in vivo, we used a SCID mouse-human xenograft model for adoptive immunotherapy. ${ }^{33}$ After intravenous infusion of a 1:1 mixture of nontransduced and iCasp $9_{\mathrm{M}}$.IRES.GFP ${ }_{\text {high }}$-transduced CTLs into SCID mice bearing an autologous LCL xenograft, mice were treated either with a single dose of CID or carrier only. Three days after CID/carrier administration, tumors were analyzed for human $\mathrm{CD}^{+} / \mathrm{GFP}^{+}$cells. Detection of the nontransduced component of the infusion product using human anti-CD3 antibodies confirmed the success of the tail-vein infusion in mice that received CID. In mice treated with CID, there was a more than $99 \%$ reduction in the number of human $\mathrm{CD}^{+} / \mathrm{GFP}^{+} \mathrm{T}$ cells, compared with infused mice treated with carrier alone, demonstrating equally high sensitivity of iCasp $9_{\mathrm{M}^{-}}$-transduced $\mathrm{T}$ cells in vivo and in vitro (Figure 7).

\section{Discussion}

A suicide gene designed to eliminate gene-modified T cells in vivo ideally should be coexpressed stably in all cells carrying the modifying gene, at levels high enough to elicit cell death. Thus, it must have low basal activity along with high specific activity, together with minimal susceptibility to endogenous antiapoptotic molecules. We have developed an inducible caspase 9 , iCasp $9_{M}$, which has low basal activity allowing stable expression for more than 4 weeks in human T cells. A single 10-nM dose of CID is sufficient to kill more than $99 \%$ of iCasp $9_{\mathrm{M}}$-transduced cells selected for high transgene expression both in vitro and in vivo. Moreover, when coexpressed with the pivotal Th1 cytokine IL-12, activation of iCasp $9_{\mathrm{M}}$ eliminated all detectable IL-12-producing cells, even without selection for high transgene expression. As caspase 9 acts downstream of most antiapoptotic molecules, a high sensitivity to CID is preserved regardless of the presence of increased levels of antiapoptotic molecules of the bcl-2 family. Thus, iCasp9 ${ }_{M}$ should induce destruction even of transformed $\mathrm{T}$ cells and memory $\mathrm{T}$ cells that are relatively resistant to apoptosis.
Recent insights into caspase 9 activation allow us to propose a molecular mechanism of iCasp $9_{\mathrm{M}}$ activation. ${ }^{34}$ In contrast to other caspase molecules, proteolysis appears to be insufficient and unnecessary for activation of caspase 9. ${ }^{35,36}$ Crystallographic and functional data indicate that dimerization of inactive caspase 9 monomers leads to conformational change-induced activation. ${ }^{37} \mathrm{In}$ a physiologic setting the concentration of pro-caspase 9 is in the 20 $\mathrm{nM}$ range, ${ }^{35}$ well below dimerization threshold. According to the proposed model, the energetic barrier to dimerization is overcome by homophilic interactions between the CARD domains of Apaf-1 and caspase 9, driven by cytochrome $\mathrm{C}$ and ATP. ${ }^{37}$ Overexpression of caspase 9 joined to 2 FKBPs results in a situation where spontaneous dimerization might occur and account for the observed toxicity of the initial construct. Removal of one FKBP resulted in increased gene expression probably by reducing spontaneous dimerization and hence toxicity. While multimerization is required for activation of surface death receptors, this model predicts that dimerization should be sufficient to mediate activation of caspase 9. Indeed, iCasp9 constructs with a single FKBP function as effectively as those with 2 FKBPs. Increased sensitivity to dimerizer by removal of the CARD domain probably represents a reduction in the energetic threshold of dimerization upon CID binding. In short, our final construct simply represents replacement of one dimerization/activation module (CARD) with another (FKBP12).

Unwanted immune responses against cells expressing virus- or bacteria-derived lethal genes such as HSV-TK and cytosine deaminase can impair their persistence..$^{5,7}$ The FKBPs and proapoptotic molecules that form the components of $\mathrm{iCasp} 9_{\mathrm{M}}$ are human-derived molecules and are therefore less likely to induce an immune response. Although the linker between FKBP and caspase 9 and the single point mutation in the FKBP domain introduce novel amino acid sequences, the latter was not immunologically recognized by macaque recipients of iFas-transduced T cells. ${ }^{15}$ Moreover, unlike virus-derived proteins such as HSV-TK, no memory T cells specific for these junctional sequences should be present, reducing the risk of immune response-mediated elimination of iCasp $9_{\mathrm{M}^{-}}$ transduced T cells.

Elimination of all cells expressing the therapeutic transgene is a prerequisite for a safety switch for clinical applications. Previous studies using inducible Fas or DED of FADD showed that approximately $10 \%$ of transduced cells were unresponsive to activation of the destructive gene. ${ }^{12,15,16}$ One explanation for unresponsiveness to CID is low expression of the transgene: both iCasp $9_{\mathrm{M}}$-transduced $\mathrm{T}$ cells in our study and iFas-transduced $\mathrm{T}$ cells in studies by others ${ }^{12,16}$ that survived after CID administration

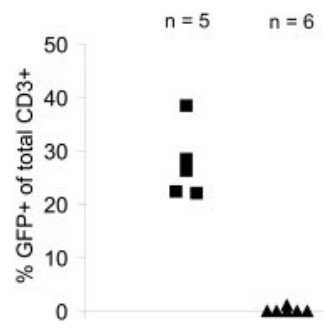

Figure 7. Function of iCasp $9_{M}$ in vivo. NOD/SCID mice were irradiated and injected subcutaneously with $10 \times 10^{6}$ to $15 \times 10^{6} \mathrm{LCLs}$. After 14 days, mice bearing tumors of $0.5 \mathrm{~cm}$ in diameter received a total of $15 \times 10^{6}$ EBV-CTLs $(50 \%$ of these cells were nontransduced and $50 \%$ were transduced with iCasp $9_{\mathrm{M}}$.I.GFP and sorted for high GFP expression). On day 3 after CTL administration, mice received either CID (50 $\mu \mathrm{g}$ AP20187; $\mathbf{\Delta}, n=6)$ or carrier only $(\boldsymbol{\square}, n=5)$ and on day 6 the presence of human $\mathrm{CD}^{+} / \mathrm{GFP}^{+} \mathrm{T}$ cells in the tumors was analyzed. Human $\mathrm{CD}^{+} \mathrm{T}$ cells isolated from the tumors of a control group of mice that received only nontransduced CTLs $\left(15 \times 10^{6}\right.$ CTLs; $\left.n=4\right)$ were used as a negative control for the analysis of $\mathrm{CD}^{+} / \mathrm{GFP}^{+} \mathrm{T}$ cells within the tumors. 
had low levels of transgene expression. We interpreted this as a retroviral "positional effect" and attempted to achieve more homogeneous expression of transgene by flanking retroviral integrants with the chicken beta-globin chromatin insulator. ${ }^{38}$ This modification dramatically increased the homogeneity of expression in transduced 293T cells, but had no significant effect in transduced primary $\mathrm{T}$ cells (data not shown). Selection of $\mathrm{T}$ cells with high expression levels minimized variability of response to the dimerizer. Over $99 \%$ of transduced $\mathrm{T}$ cells sorted for high GFP expression were eliminated after a single 10-nM CID dose. This demonstration supports the hypothesis that cells expressing high levels of suicide gene can be isolated using a selectable marker. Although a very small number of residual cells may cause resurgence of toxicity, a deletion efficiency of up to 2 logs will significantly decrease this possibility. For clinical use, coexpression with a nonimmunogenic selectable marker such as truncated human NGFR, CD20, or CD34 instead of GFP will allow for selection of high transgene-expressing T cells. ${ }^{39-41}$ Coexpression of such a selectable marker can be obtained using either an IRES or posttranslational modification of a fusion protein containing a self-cleaving (eg, 2A) sequence. ${ }^{42}$ In contrast, in situations where the sole safety concern is the transgene-mediated (eg, artificial T-cell receptors, cytokines) toxicity, this selection step may be unnecessary, as tight linkage between $i \mathrm{Casp} 9_{\mathrm{M}}$ and transgene expression ensures elimination of those cells that are expressing biologically relevant levels of the therapeutic transgene. We demonstrated this by coexpressing iCasp $9_{M}$ with IL-12; activation of iCasp $9_{M}$ completely abolished measurable IL-12 production. However, this may depend on the function and the activity of the transgene.

The other explanation for unresponsiveness to CID is that high levels of apoptosis inhibitors may attenuate CID-mediated apoptosis. These include c-FLIP, bcl-2 family members, and inhibitors of apoptosis proteins (IAPs), which normally regulate the balance between apoptosis and survival. For instance, upregulation of
c-FLIP and bcl-2 render a subpopulation of $\mathrm{T}$ cells, destined to establish the memory pool, resistant to activation-induced cell death in response to cognate target or antigen-presenting cells. ${ }^{18,30}$ In several T-lymphoid tumors, the physiologic balance between apoptosis and survival is disrupted in favor of cell survival. ${ }^{28,29} \mathrm{~A}$ suicide gene should delete all transduced T cells including memory and malignantly transformed cells. Thus, to ensure safety, preserved function of the inducible suicide gene in the presence of increased levels of antiapoptotic molecules is critical. The apical location of iFas (or iFADD) in the apoptosis signaling pathway may leave it especially vulnerable to inhibitors of apoptosis and these molecules are therefore less suited to being the key component of an apoptotic safety switch. While caspase 3 or 7 as terminal effector molecules appear to be ideal candidates, we were unable to express either in primary human $\mathrm{T}$ cells at functional levels (data not shown). One possible explanation is that caspase 3 and 7 , unlike caspase 9, make poor substrates for themselves and thus require prohibitively high cellular concentrations for cleavage. ${ }^{14}$ We therefore chose caspase 9 , which bypasses the inhibitory effects of c-FLIP and antiapoptotic bcl-2 family members and could be expressed stably at functional levels. Although X-linked inhibitor of apoptosis (XIAP) could in theory reduce spontaneous caspase 9 activation (Figure 1), ${ }^{43}$ the high affinity of AP20187 (or AP1903) for $\mathrm{FKBP}_{\mathrm{V} 36}$ likely displaces this noncovalently associated XIAP. Indeed, in contrast to iFas, iCasp $9_{M}$ remained functional in a transformed T-cell line that overexpresses antiapoptotic molecules, including bcl- $\mathrm{x}_{\mathrm{L}}$.

We have described a new inducible safety switch, designed specifically for expression from an oncoretroviral vector by human T cells. iCasp9 $9_{M}$ can be activated by AP1903 (or analogs), a small chemical inducer of dimerization that has proven safe at the required dose for optimum deletional effect, ${ }^{11}$ and unlike ganciclovir or rituximab has no other biologic effects in vivo. Therefore, expression of this suicide gene in T cells for adoptive transfer will increase safety and hence broaden the scope of clinical applications.

\section{References}

1. Walter EA, Greenberg PD, Gilbert MJ, et al. Reconstitution of cellular immunity against cytomegalovirus in recipients of allogeneic bone marrow by transfer of T-cell clones from the donor. $\mathrm{N} \mathrm{Engl}$ J Med. 1995;333:1038-1044.

2. Rooney $\mathrm{CM}$, Smith $\mathrm{CA}, \mathrm{Ng} \mathrm{CY}$, et al. Infusion of cytotoxic $T$ cells for the prevention and treatment of Epstein-Barr virus-induced lymphoma in allogeneic transplant recipients. Blood. 1998;92: 1549-1555.

3. Dudley ME, Wunderlich JR, Robbins PF, et al. Cancer regression and autoimmunity in patients after clonal repopulation with antitumor lymphocytes. Science. 2002;298:850-854.

4. Marijt WA, Heemskerk MH, Kloosterboer FM, et al. Hematopoiesis-restricted minor histocompatibility antigens HA-1- or HA-2-specific T cells can induce complete remissions of relapsed leukemia. Proc Natl Acad Sci U S A. 2003;100:27422747.

5. Bonini C, Ferrari G, Verzeletti S, et al. HSV-TK gene transfer into donor lymphocytes for control of allogeneic graft-versus-leukemia. Science. 1997;276:1719-1724.

6. Tiberghien P, Ferrand C, Lioure B, et al. Administration of herpes simplex-thymidine kinaseexpressing donor T cells with a T-cell-depleted allogeneic marrow graft. Blood. 2001;97:63-72.

7. Riddell SR, Elliott M, Lewinsohn DA, et al. T-cell mediated rejection of gene-modified HIV-specific cytotoxic T lymphocytes in HIV-infected patients. Nat Med. 1996;2:216-223.
8. Freytag SO, Khil M, Stricker H, et al. Phase I study of replication-competent adenovirusmediated double suicide gene therapy for the treatment of locally recurrent prostate cancer. Cancer Res. 2002;62:4968-4976.

9. Introna M, Barbui AM, Bambacioni F, et al. Genetic modification of human T cells with CD20: a strategy to purify and lyse transduced cells with anti-CD20 antibodies. Hum Gene Ther. 2000;11: 611-620.

10. Clackson T, Yang W, Rozamus LW, et al. Redesigning an FKBP-ligand interface to generate chemical dimerizers with novel specificity. Proc Natl Acad Sci U S A. 1998;95:10437-10442.

11. Iuliucci JD, Oliver SD, Morley S, et al. Intravenous safety and pharmacokinetics of a novel dimerizer drug, AP1903, in healthy volunteers. J Clin Pharmacol. 2001;41:870-879.

12. Thomis DC, Marktel S, Bonini C, et al. A Fasbased suicide switch in human T cells for the treatment of graft-versus-host disease. Blood. 2001;97:1249-1257.

13. Spencer DM, Belshaw PJ, Chen L, et al. Functional analysis of Fas signaling in vivo using synthetic inducers of dimerization. Curr Biol. 1996;6: 839-847.

14. Fan L, Freeman KW, Khan T, Pham E, Spencer DM. Improved artificial death switches based on caspases and FADD. Hum Gene Ther. 1999;10: 2273-2285.

15. Berger C, Blau CA, Huang ML, et al. Pharmacologically regulated Fas-mediated death of adop- tively transferred T cells in a nonhuman primate model. Blood. 2004;103:1261-1269.

16. Junker K, Koehl U, Zimmerman S, et al. Kinetics of cell death in T lymphocytes genetically modified with two novel suicide fusion genes. Gene Ther. 2003;10:1189-1197.

17. Yu J, Zhang L. Apoptosis in human cancer cells. Curr Opin Oncol. 2004;16:19-24.

18. Grayson JM, Zajac AJ, Altman JD, Ahmed R. Cutting edge: increased expression of $\mathrm{Bcl}-2$ in antigen-specific memory CD8 ${ }^{+} \mathrm{T}$ cells. J Immunol. 2000;164:3950-3954.

19. Bollard CM, Rossig C, Calonge MJ, et al. Adapting a transforming growth factor beta-related tumor protection strategy to enhance antitumor immunity. Blood. 2002;99:3179-3187.

20. Wagner HJ, Bollard CM, Vigouroux S, et al. A strategy for treatment of Epstein-Barr viruspositive Hodgkin's disease by targeting interleukin 12 to the tumor environment using tumor antigen-specific T cells. Cancer Gene Ther. 2004;11: 81-91.

21. Pule M, Finney H, Lawson A. Artificial T-cell receptors. Cytotherapy. 2003;5:211-226.

22. Schumacher TN. T-cell-receptor gene therapy. Nat Rev Immunol. 2002;2:512-519.

23. Anderson R, Macdonald I, Corbett T, et al. Construction and biological characterization of an interleukin-12 fusion protein (Flexi-12): delivery to acute myeloid leukemic blasts using adenoassociated virus. Hum Gene Ther. 1997;8:11251135. 
From www.bloodjournal.org at UCL Library Services on September 16, 2008. For personal use only.

24. Cosset FL, Takeuchi Y, Battini JL, Weiss RA, Collins MK. High-titer packaging cells producing recombinant retroviruses resistant to human serum. J Virol. 1995;69:7430-7436.

25. Smith $\mathrm{CA}, \mathrm{Ng} \mathrm{CY}$, Heslop $\mathrm{HE}$, et al. Production of genetically modified Epstein-Barr virus-specific cytotoxic T cells for adoptive transfer to patients at high risk of EBV-associated lymphoproliferative disease. J Hematother. 1995;4:73-79.

26. Hawley RG, Hawley TS, Fong AZ, et al. Thrombopoietic potential and serial repopulating ability of murine hematopoietic stem cells constitutively expressing interleukin 11. Proc Natl Acad Sci U S A. 1996;93:10297-10302.

27. Gottschalk S, Heslop HE, Roon CM. Treatment of Epstein-Barr virus-associated malignancies with specific T cells. Adv Cancer Res. 2002;84:175201.

28. Schattner EJ. Apoptosis in lymphocytic leukemias and lymphomas. Cancer Invest. 2002;20:737748.

29. Nakatsuka S, Takakuwa T, Tomita Y, et al. Hypermethylation of death-associated protein (DAP) kinase $\mathrm{CpG}$ island is frequent not only in B-cell but also in T- and natural killer (NK)/T-cell malignancies. Cancer Sci. 2003;94:87-91.

30. Kirchhoff S, Muller WW, Li-Weber M, Krammer $\mathrm{PH}$. Up-regulation of c-FLIPshort and reduction of activation-induced cell death in CD28-costimu- lated human T cells. Eur J Immunol. 2000;30: 2765-2774.

31. Zehender G, Varchetta S, De Maddalena C, et al Resistance to Fas-mediated apoptosis of human T-cell lines expressing human T-lymphotropic virus type-2 (HTLV-2) Tax protein. Virology. 2001; 281:43-50.

32. Kongphanich A, Hieda M, Kurokawa K, Murata T, Kobayashi N. Overcoming the blockade at the upstream of caspase cascade in Fas-resistant HTLV-I-infected T cells by cycloheximide. Biochem Biophys Res Commun. 2002;294:714-718.

33. Lacerda JF, Ladanyi M, Louie DC, et al. Human Epstein-Barr virus (EBV)-specific cytotoxic T lymphocytes home preferentially to and induce selective regressions of autologous EBV-induced $B$ cell lymphoproliferations in xenografted C.B-17 scid/scid mice. J Exp Med. 1996;183:1215-1228.

34. Boatright KM, Salvesen GS. Mechanisms of caspase activation. Curr Opin Cell Biol. 2003;15: 725-731.

35. Stennicke HR, Deveraux QL, Humke EW, et al. Caspase- 9 can be activated without proteolytic processing. J Biol Chem. 1999;274:8359-8362.

36. Boatright KM, Renatus M, Scott FL, et al. A unified model for apical caspase activation. Mol Cell 2003;11:529-541.

37. Renatus M, Stennicke HR, Scott FL, Liddington
RC, Salvesen GS. Dimer formation drives the activation of the cell death protease caspase 9 . Proc Natl Acad Sci U S A. 2001;98:14250-14255.

38. Emery DW, Yannaki E, Tubb J, Stamatoyannopoulos G. A chromatin insulator protects retrovirus vectors from chromosomal position effects. Proc Natl Acad Sci U S A. 2000;97:9150-9155

39. Deola S, Scaramuzza S, Birolo RS, et al. Mobilized blood CD34 ${ }^{+}$cells transduced and selected with a clinically applicable protocol reconstitute lymphopoiesis in SCID-Hu mice. Hum Gene Ther. 2004;15:305-311.

40. Fehse B, Kustikova OS, Li Z, et al. A novel 'sortsuicide' fusion gene vector for $T$ cell manipulation. Gene Ther. 2002;9:1633-1638.

41. Serafini M, Manganini M, Borleri G, et al. Characterization of CD20-transduced T lymphocytes as an alternative suicide gene therapy approach for the treatment of graft-versus-host disease. Hum Gene Ther. 2004;15:63-76

42. Szymczak AL, Workman CJ, Wang Y, et al. Correction of multi-gene deficiency in vivo using a single 'self-cleaving' $2 \mathrm{~A}$ peptide-based retroviral vector. Nat Biotechnol. 2004;22:589-594.

43. Srinivasula SM, Hegde R, Saleh A, et al. A conserved XIAP-interaction motif in caspase-9 and Smac/DIABLO regulates caspase activity and apoptosis. Nature. 2001;410:112-116. 